\title{
Short communication: Bovine leukemia virus infection in adult cows does not interfere with foot-and-mouth disease vaccination
}

\author{
Juan Pablo Jaworski, ${ }^{*} \dagger^{1}$ Juan Manuel Sala, ${ }^{, 1}$ and Alejandra Capozzo† ${ }^{2}$ \\ *Instituto Nacional de Tecnología Agropecuaria, Instituto de Virología, Buenos Aires, 1686, Argentina \\ †Consejo Nacional de Investigaciones Científicas y Técnicas, Buenos Aires, 1425, Argentina
}

\begin{abstract}
Bovine leukemia virus (BLV) is a widespread infection that can affect innate and adaptive immunity; however, little information exists on how BLV infection affects foot-and-mouth disease virus (FMDV) vaccination programs. Vaccination for FMDV is compulsory in many regions of the world, and vaccine efficacy is monitored by measuring total antibodies against this virus. In a previous study, we observed that BLV-infected heifers produced a lower amount of antibodies in response to FMDV at first vaccination. In this followup study, we show that BLV status does not interfere with the total level and avidity of anti-FMDV-specific antibodies induced after repetitive routine vaccination in adult cattle. This is relevant information for the proficiency of vaccine-based FMDV control programs in BLV-endemic regions.
\end{abstract}

Key words: bovine leukemia virus, foot-and-mouth disease, vaccination program, herd immunity

\section{Short Communication}

Bovine leukemia virus (BLV) is the causative agent of enzootic bovine leucosis. In one third of infected animals BLV infection progresses to a state of persistent lymphocytosis, and in about 1 to $5 \%$ of infected animals it develops lymphosarcoma (Ghysdael et al., 1984). Bovine leukemia virus is endemic worldwide, except for western Europe and Oceania. In the United States, more than $83 \%$ of dairy herds are infected with BLV and the within-herd prevalence is about $50 \%$, on average (Bartlett et al., 2014). In the main dairy-producing region of Argentina, the within-herd prevalence of BLV is close to $80 \%$, on average, and more than $95 \%$ of herds are infected with BLV (Gutiérrez et al., 2012).

Received April 13, 2018.

Accepted August 5, 2018.

${ }^{1}$ Both authors contributed equally.

${ }^{2}$ Corresponding author: capozzo.alejandra@inta.gob.ar
High prevalence of BLV is associated with a significant economic loss on dairy industry. (Bartlett et al., 2013). Recent studies suggested that BLV infection might negatively affect animal immunity (Frie and Coussens, 2015). In this regard, 3 separate studies showed that immunological disorders provoked during BLV natural infection may counteract the development of immunity after commercial and experimental vaccination (Erskine et al., 2011; Frie et al., 2016; Puentes et al., 2016; Frie et al., 2017). Considering that BLV is endemic in many countries and approximately 50 to $70 \%$ of animals are asymptomatic, it is important to know whether BLV infection interferes with the immunogenicity elicited by those vaccines typically used in bovines, particularly those that are part of national programs.

Vaccine-based control programs are applied worldwide to combat foot-and-mouth disease. Foot-and-mouth disease is a highly contagious acute vesicular viral disease that affects cloven-hoofed animals, causing death only in young animals and high morbidity in adults The circulation of foot-and-mouth disease virus (FMDV) in susceptible livestock imposes severe restrictions on the movement and trade of animals and derived products, causing serious economic loss to the affected countries (Smith et al., 2014). Foot-and-mouth disease virus is endemic in many parts of Asia, Africa, and South America, where vaccination of susceptible populations is widely used as a major control measure. Argentina is free from the disease and has 2 epidemiological regions: (1) the southern region without vaccination and (2) the central and northern regions, where the cattle population is vaccinated systematically, either once or twice per year (animals $>2$ and $<2$ yr of age, respectively). The commercial vaccine is composed of oil-adjuvanted suspensions of inactivated viral particles belonging to the strains A24/Cruzeiro, A/Argentina/2001, O1/ Campos, and C3/Indaial. Vaccine-induced protection is mediated by specific antibodies, and maintaining high levels of total antibodies against FMDV is paramount to prevent outbreaks, keeping the World Organisation for Animal Health (OIE) free-with-vaccination status and, thus, the international markets. The level 
of FMDV antibodies measured liquid-phase blocking ELISA (LPBE) has been widely used to evaluate the vaccination efficacy (Robiolo et al., 1995; Mattion et al., 2004). In this regard, several curves relating LPBE titers have been published with estimated protection percentages (EPP; Maradei et al., 2008; Robiolo et al., 2010). Particular LPBE titers have been correlated with an EPP of $75 \%$ for the different FMDV strains (i.e., A24/Cruzeiro $\log _{10}$ titer $=1.90$, and O1/Campos $\log _{10}$ titer $=2.11$ ).

To date, scarce information exists on how BLV infection might interfere with the immunity induced after FMDV vaccination and affect the epidemiological scenario of vaccine efficacy in the control area. In a previous study, we showed that BLV-infected heifers produced lower IgM and $\operatorname{IgG}_{1}$ titers in response to the first FMDV vaccination (Puentes et al., 2016). In the present study, we sought to determine if the BLV status of cattle interfered with the antibody production in response to repetitive vaccination with a commercial tetravalent FMDV vaccine. In addition, we measured the avidity of antibodies as an indicator of affinity maturation and B-cell memory induced through FMDV vaccination in BLV-infected versus not infected cattle.

To investigate whether BLV natural infection may interfere with FMDV vaccination, we worked with 2 separate dairy herds with markedly different BLV status. The high-prevalence herd belonged to a dairy farm located in Rafaela, Santa Fe, Argentina, and the low-prevalence herd belonged to a different facility located in Brandsen, Buenos Aires, Argentina. Both farms were composed of approximately 500 Holstein animals, including the milking herd, dry cows, heifers, and calves. The BLV serological status of all animals in both farms was assessed at the time the study was initiated. Plasma-specific antibodies against the whole BLV viral particle were measured by indirect ELISA, as described previously (Trono et al., 2001). Normalized results were obtained as a sample-to-positive ratio. A weak positive control serum was used to calculate the ratio; its reactivity was set to $100 \%$ and all tested samples were referred to the control. A cutoff level of $25 \%$ was established and those samples with reactivity above the cutoff level were considered positive. The within-herd BLV prevalence of the high- and lowprevalence farms was 90 and 5\%, respectively. The low BLV seroprevalence for the second farm was explained by specific control measures applied in this particular farm to reduce BLV prevalence.

For this particular study, 200 milking cows (>2 yr) were selected from both farms based on their BLV serologic status; 100 positive $(\mathbf{B L V}+)$ and 100 negative (BLV - ) cows were selected from the high- and lowprevalence herds, respectively. All animals included in the present study had been vaccinated with a FMDV commercial vaccine at least 4 times (including 1 prime and more than 3 boosters) as part of the official footand-mouth disease national campaign. Serum samples were collected from these animals to measure the level of FMDV-specific antibodies as an indicator of vaccine-induced immunity (Maradei et al., 2008). The procedures used for animal handling and sampling were approved by the Institutional Animal Care and Use Committee of Instituto Nacional de Tecnología Agropecuaria (INTA, Buenos Aires, Argentina). The guidelines (https://inta.gob.ar/documentos/leyes-decretos -disposiciones) described in the institutional manual were followed at all times.

Total serum antibody responses against FMDV O1/ Campos and A24/Cruzeiro strains were assessed by LPBE performed as stated by the OIE Manual (Bucafusco et al., 2014). Briefly, a rabbit antiserum was used to capture inactivated whole 140S FMDV particles. Strains of O1/Campos or A24/Cruzeiro FMDV were used because they were the same strains included in the commercial tetravalent vaccine. Antibody titers were expressed as the reciprocal $\log 10$ of serum dilutions, giving $50 \%$ of the absorbance recorded in the virus control wells without serum. Individual antibody levels against O1/Campos and A24/Cruzeiro FMDV strains in BLV + and BLV - animals are shown in Figure 1A and B. No differences in FMDV antibody titers (either O1/Campos or A24/Cruzeiro) were observed between animals from the high- and low-BLV prevalence herds. Except for a couple of BLV+ animals that showed low anti-FMDV O1/Campos antibody titers, all the remaining animals from both herds had anti-FMDV antibody titers well above the threshold associated with an EPP of $75 \%$, indicating a high herd immunity level in from both groups (Maradei et al., 2008). This observation suggests that the BLV status would not interfere with the efficacy of the FMDV revaccination in cattle.

Although our study was not designed to address a particular immune impairment due to BLV infection, we investigated if the BLV status in cattle would modulate the affinity maturation process induced after repetitive vaccination ( $>3$ boosters). For that purpose, we measured the avidity of antibodies as an indicator of affinity maturation and B-cell memory induced by FMDV vaccination in 80 randomly selected BLV+ and BLV - animals ( $\mathrm{n}=40$ for each group; Lavoria et al., 2012). The avidity index (AvI) of anti-A24/Cruzeiroand O1/Campos-specific antibodies was estimated as described before (Lavoria et al., 2012). Briefly, the AvI was calculated as the percentage of residual activity of the serum sample diluted 1:50 after a 20-min urea washing step, relative to that of untreated sample: $\mathrm{AvI} \%=(\mathrm{OD}$ sample with urea/OD sample without 
urea) $\times 100$, where OD $=$ optical density. Interestingly, we observed no significant differences in the AvI of antibodies from BLV+ or BLV - animals (Figure 1CD). Mean AvI values were above $60 \%$ for both groups and for both viruses, similar to those reported before in cattle after 2 vaccinations using the same vaccines (Lavoria et al., 2012). These results suggest that BLV status did not affect the development of B-cell memory compartment and the affinity maturation process induced by repetitive FMDV vaccination in cattle.

Previous studies showed that BLV infection might negatively affect animal immunity elicited in response to commercial and experimental vaccines. In a previous study from our group, we demonstrated that BLV+ 10-mo-old heifers produced lower IgM and $\operatorname{IgG}_{1}$ titers in response to the first FMDV vaccination (Puentes et al., 2016). The lower level of anti-FMDV- $\operatorname{IgG}_{1}$ titers in $\mathrm{BLV}+$ animals was transient, only noticeable at $2 \mathrm{wk}$ after vaccination. In contrast, total FMDV antibody and $\mathrm{IgG}_{2}$ titers were unaffected by the BLV status. Supporting these observations, other groups described altered patterns of antibody production after first vaccination in BLV+ dairy cows (Erskine et al., 2011; Frie et al., 2016).

In another series of studies, the authors suggested that the alteration in antibody production could be due to abnormalities in both B- and T-cell subsets also described in BLV+ cows (Frie et al., 2016, 2017). Different outcomes observed in these studies, regarding which particular immune component was affected by BLV infection, might be explained either (1) the vaccine composition (antigen/immunogen), (2) the type of pathogens targeted by the vaccine (virus/bacteria), (3) the physiological status of the animals, (4) the relationship between the occurrence of BLV infection and the timing of vaccination (prime/boost), or (5) the number and timing of booster vaccination. All these parameters should be further explored.
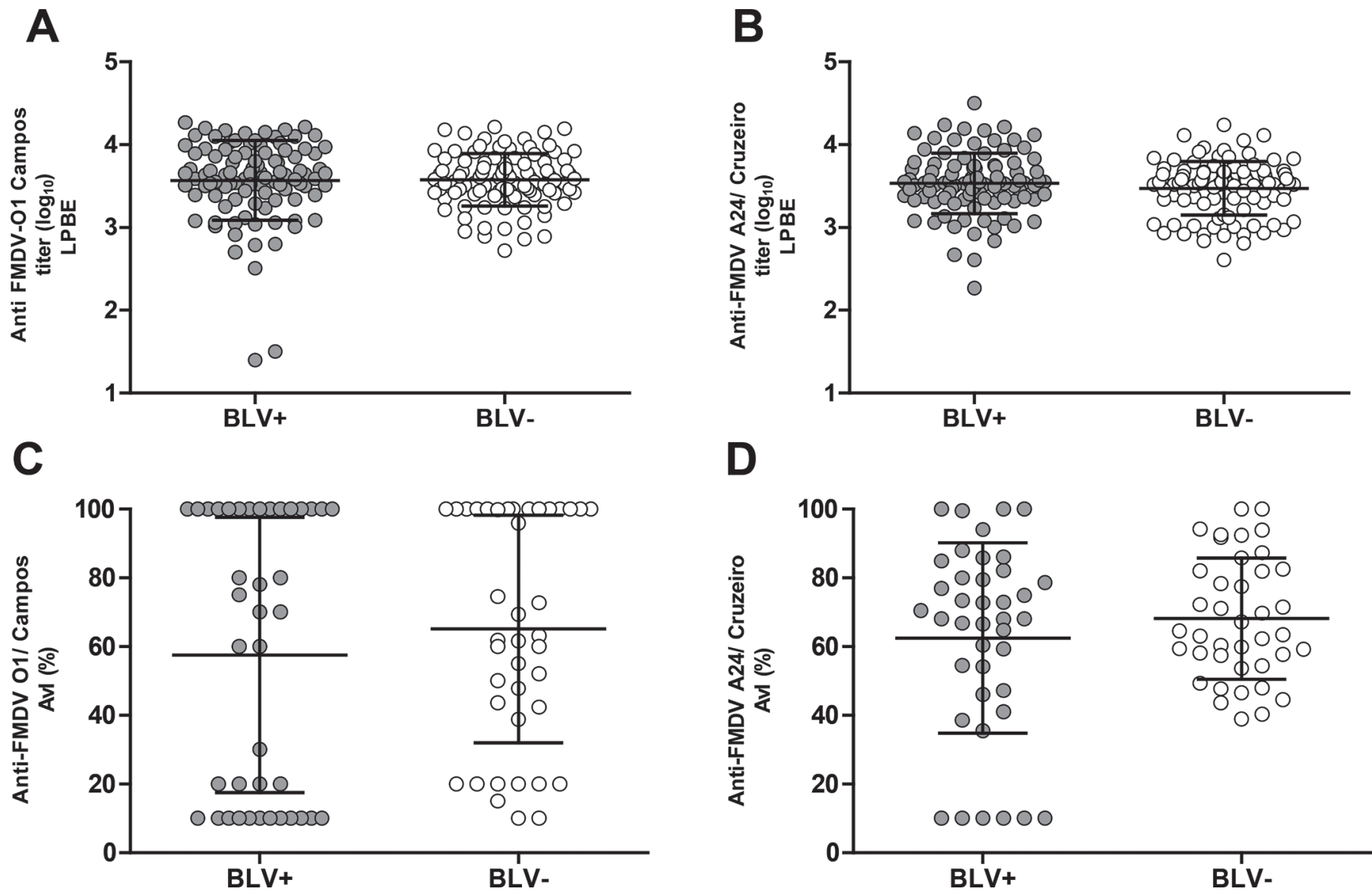

Figure 1. Antibody production in response to foot-and-mouth disease virus (FMDV) vaccination in bovine leukemia virus-infected (BLV+) and not infected (BLV-) cattle. Total antibody titers against O1/Campos (A) and A24/Cruzeiro (B) FMDV strains measured by liquid phase blocking ELISA (LPBE). Avidity index (AvI) of O1/Campos- (C) and A24/Cruzeiro-specific (D) antibodies measured by ELISA. Mean titers and AvI as well as SE are depicted. All BLV+ and BLV- animals received 4 immunizations with a tetravalent FMDV commercial vaccine. No significant differences $(P<0.05)$ were observed between BLV+ and BLV- cattle in both assays. 
The aim of our study was to investigate whether BLV status could interfere with the efficacy of the FMDV vaccination campaign. This is of particular interest in FMDV-endemic regions, as all FMDV antibody titers induced through vaccination are necessary to prevent disease outbreaks. Here we showed that, after repeated vaccination, levels and avidity of anti-FMDV antibodies were similar between BLV+ and BLV - animals. Although first vaccination may be affected (Puentes et al., 2016), repeated vaccination probably weakens this effect at the herd level, as animals may be infected with BLV at different times before or after their first vaccination. As a whole, our results suggest that BLV status does not compromise the efficacy of routine FMDV vaccination in cattle.

\section{ACKNOWLEDGMENTS}

This study was supported by INTA (Instituto Nacional de Tecnología Agropecuaria Argentina, Buenos Aires, Argentina) through PNSA-1115053 and PNSA1115052). JPJ, KT and AVC are researchers of CONICET (Consejo Nacional de Investigaciones Científicas y Técnicas, Buenos Aires, Argentina).

\section{REFERENCES}

Bartlett, P. C., B. Norby, T. M. Byrem, A. Parmelee, J. T. Ledergerber, and R. J. Erskine. 2013. Bovine leukemia virus and cow longevity in Michigan dairy herds. J. Dairy Sci. 96:1591-1597.

Bartlett, P. C., L. M. Sordillo, T. M. Byrem, B. Norby, D. L. Grooms, C. L. Swenson, J. Zalucha, and R. J. Erskine. 2014. Options for the control of bovine leukemia virus in dairy cattle. J. Am. Vet. Med. Assoc. 244:914-922.

Bucafusco, D., S. Di Giacomo, J. Pega, M. S. Juncos, J. M. Schammas, M. Perez-Filgueira, and A. V. Capozzo. 2014. Influence of antibodies transferred by colostrum in the immune responses of calves to current foot-and-mouth disease vaccines. Vaccine 32:6576-6582.

Erskine, R. J., P. C. Bartlett, K. M. Sabo, and L. M. Sordillo. 2011. Bovine leukemia virus infection in dairy cattle: Effect on serological response to immunization against J5 Escherichia coli bacterin. Vet. Med. Int. 2011:915747.

Frie, M. C., and P. M. Coussens. 2015. Bovine leukemia virus: A major silent threat to proper immune responses in cattle. Vet. Immunol. Immunopathol. 163:103-114.

Frie, M. C., K. R. Sporer, J. C. Wallace, R. K. Maes, L. M. Sordillo, P. C. Bartlett, and P. M. Coussens. 2016. Reduced humoral im- munity and atypical cell-mediated immunity in response to vaccination in cows naturally infected with bovine leukemia virus. Vet. Immunol. Immunopathol. 182:125-135.

Frie, M. C., K. R. B. Sporer, O. J. Benitez, J. C. Wallace, C. J. Droscha, P. C. Bartlett, and P. M. Coussens. 2017. Dairy cows naturally infected with bovine leukemia virus exhibit abnormal B- and T-cell phenotypes after primary and secondary exposures to keyhole limpet hemocyanin. Front. Vet. Sci. 4:112.

Ghysdael, J., C. Bruck, R. Kettmann, and A. Burny. 1984. Bovine leukemia virus. Curr. Top. Microbiol. Immunol. 112:1-19.

Gutiérrez, G., H. Carignano, I. Alvarez, C. Martinez, N. Porta, R. Politzki, M. Gammella, M. Lomonaco, N. Fondevila, M. Poli, and K. Trono. 2012. Bovine leukemia virus p24 antibodies reflect blood proviral load. BMC Vet. Res. 8:187.

Lavoria, M. A., S. Di-Giacomo, D. Bucafusco, O. L. Franco-Mahecha, D. M. Perez-Filgueira, and A. V. Capozzo. 2012. Avidity and subtyping of specific antibodies applied to the indirect assessment of heterologous protection against foot-and-mouth disease virus in cattle. Vaccine 30:6845-6850.

Maradei, E., J. La Torre, B. Robiolo, J. Esteves, C. Seki, A. Pedemonte, M. Iglesias, R. D'Aloia, and N. Mattion. 2008. Updating of the correlation between lpELISA titers and protection from virus challenge for the assessment of the potency of polyvalent aphtovirus vaccines in Argentina. Vaccine 26:6577-6586.

Mattion, N., G. Konig, C. Seki, E. Smitsaart, E. Maradei, B. Robiolo, S. Duffy, E. Leon, M. Piccone, A. Sadir, R. Bottini, B. Cosentino, A. Falczuk, R. Maresca, O. Periolo, R. Bellinzoni, A. Espinoza, J. L. Torre, and E. L. Palma. 2004. Reintroduction of foot-andmouth disease in Argentina: Characterisation of the isolates and development of tools for the control and eradication of the disease. Vaccine 22:4149-4162.

Puentes, R., L. De Brun, A. Algorta, V. Da Silva, F. Mansilla, G. Sacco, S. Llambi, and A. V. Capozzo. 2016. Evaluation of serological response to foot-and-mouth disease vaccination in BLV infected cows. BMC Vet. Res. 12:119.

Robiolo, B., P. R. Grigera, O. H. Periolo, C. Seki, T. Bianchi, E Maradei, and J. L. La Torre. 1995. Assessment of foot and mouth disease vaccine potency by liquid-phase blocking ELISA: A proposal for an alternative to the challenge procedure in Argentina. Vaccine 13:1346-1352.

Robiolo, B., J. La Torre, E. Maradei, C. P. Beascoechea, A. Perez, C. Seki, E. Smitsaart, N. Fondevila, E. Palma, N. Goris, K. De Clercq, and N. Mattion. 2010. Confidence in indirect assessment of foot-and-mouth disease vaccine potency and vaccine matching carried out by liquid phase ELISA and virus neutralization tests. Vaccine 28:6235-6241.

Smith, M. T., A. M. Bennett, M. J. Grubman, and B. C. Bundy. 2014. Foot-and-mouth disease: technical and political challenges to eradication. Vaccine 32:3902-3908.

Trono, K. G., D. M. Perez-Filgueira, S. Duffy, M. V. Borca, and C. Carrillo. 2001. Seroprevalence of bovine leukemia virus in dairy cattle in Argentina: comparison of sensitivity and specificity of different detection methods. Vet. Microbiol. 83:235-248. 